\title{
CARCINOMA OF THE BRONCHUS PRESENTING AS THIN-WALLED CYSTS
}

\author{
BY \\ H. J. ANDERSON AND J. W. PIERCE \\ From St. Thomas's Hospital, London
}

(RECEIVED FOR PUbLICATION OCTOBER 4, 1953)

Our object in this paper is to draw attention to an uncommon manifestation of bronchial carcinoma which we believe is not widely recognized, and to suggest a possible mechanism for its production.

During the past five years we have encountered six cases of carcinoma of the bronchus in which the radiological appearance was that of a thin-walled cavity or cyst. These cavities have no special radiological characteristics by which they can be distinguished with any certainty from non-malignant conditions, but they differ in several respects from the appearances commonly produced when a solid neoplasm breaks down.

The most notable feature of these cavities is that the walls are thin, in some cases very thin, with a smooth and regular internal surface and a fairly clear-cut outer margin. They have been of different sizes, from 1 to 3 in. in diameter, the smaller roughly circular, the larger examples oval or irregular. They are not surrounded by any consolidation, and in none has there been a fluid level. Some appear to be in free communication with a bronchus, as in one case the cavity changed shape and size during respiration (Fig. 7), and another became gradually smaller over a period of two months (Figs. 4 and 5).

Their growth is slow and appears to be a simple expansion; it certainly does not involve a spreading consolidation which later excavates. The hilar glands may enlarge later in the illness, and terminally the radiograph may be complicated by inflammatory consolidation. The clinical features, course, duration, and prognosis of this type of neoplasm do not appear to differ from those of the ordinary peripheral bronchial carcinoma.

\section{Morbid ANatomy and Histology}

Examination of the specimens removed at operation or necropsy shows a cavity in the lungusually empty - with a lining in some parts smooth, grey, and shining, but in parts where there is superficial inflammation rough and opaque. The cavity wall is composed of a mixture of fibrous tissue and well-differentiated squamous carcinoma cells, and the inner surface of the cavity may con- -5 sist of these malignant cells, or of flattened or squamous metaplastic epithelium, with the malignant tissue spreading just beneath the surface (Fig.. 10). The malignant tissue may be very thin, in $\overrightarrow{0}$ one case only two or three cells in depth, and parts of the cavity wall may be formed of compressed lung only; elsewhere there may be a thin coverings of inflammatory products. In two of our cases a small nodule composed of growth and fibrous tissuei has been present at the junction of the bronchuso and the cavity (Figs. 8, 9, and 11), and it is possible that this could have produced an intermittento valvular bronchial occlusion; but of this we have no proof.

There is no evidence of any extension of the malignant tissue into the surrounding lung, though there may be a few radiating strands of fibrouso tissue around the cavity, nor of degeneration of necrosis of the malignant cells in the cavity wall

\section{Discussion}

We do not believe that these cavities are formedo by the breaking down of a solid neoplasm, first, $>$ because their thin, smooth walls are so differento from the thick-walled, irregular abscess which usually results when a peripheral carcinoma exca- 0 vates; secondly, because we have never observed $N$ a solid growth actually breaking down into this type of cavity; and thirdly, because one would noto expect to find non-malignant epithelium lining the wall of a broken-down solid neoplasm.

We suggest as an alternative and more probable $\stackrel{\infty}{+}$ mechanism that this variety of bronchial carcinoma may arise when a thin layer of malignant cellso

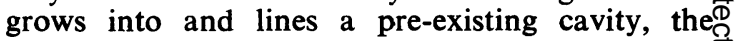
cavity being formed either by pressure changes $\mathbb{Q}$ consequent on a valvular obstruction to a bronchus by a small nodule of growth, or by inflammatory changes in the proximity of but not involving theo neoplasm. This hypothesis is supported by the 


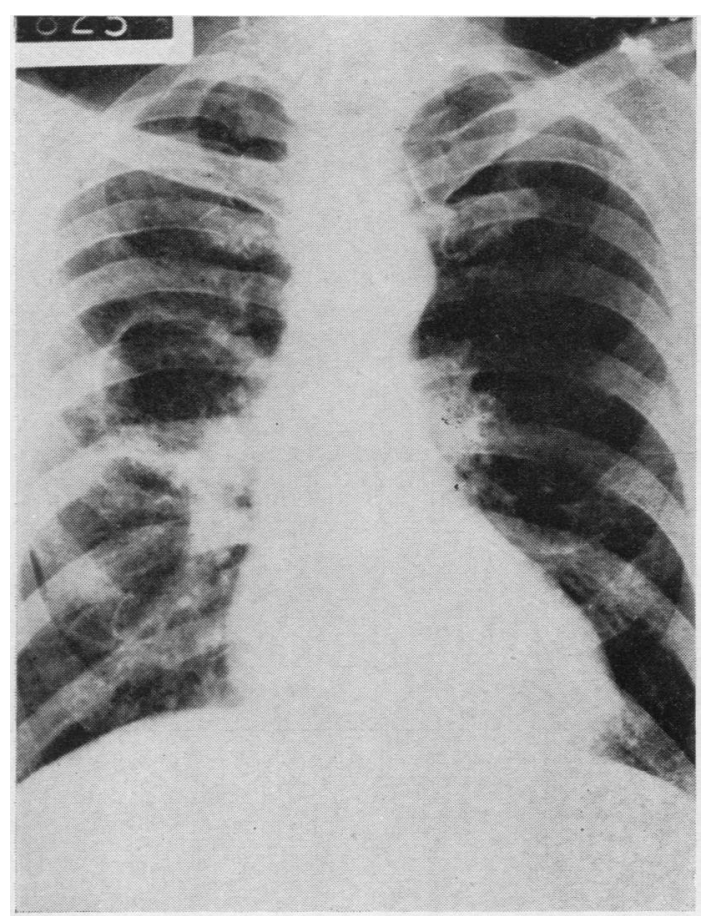

Fig. 1a.-Case 1: thin-walled cavity in right upper lobe in January, 1948.

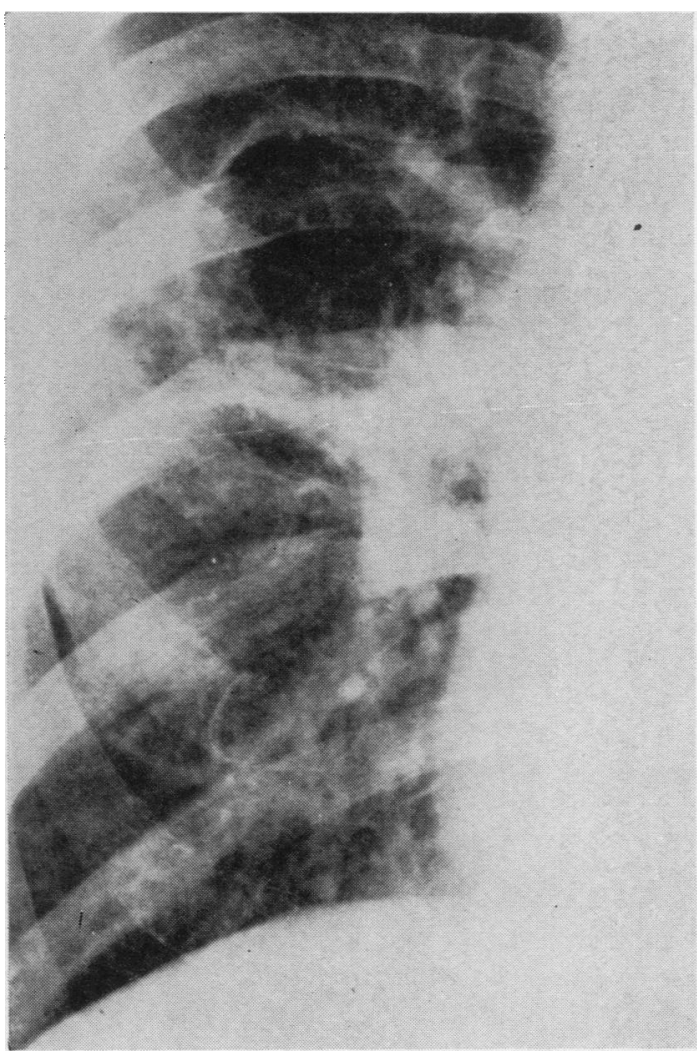

FIG. 1b.-Case 1: detailed view of Fig. 1a.

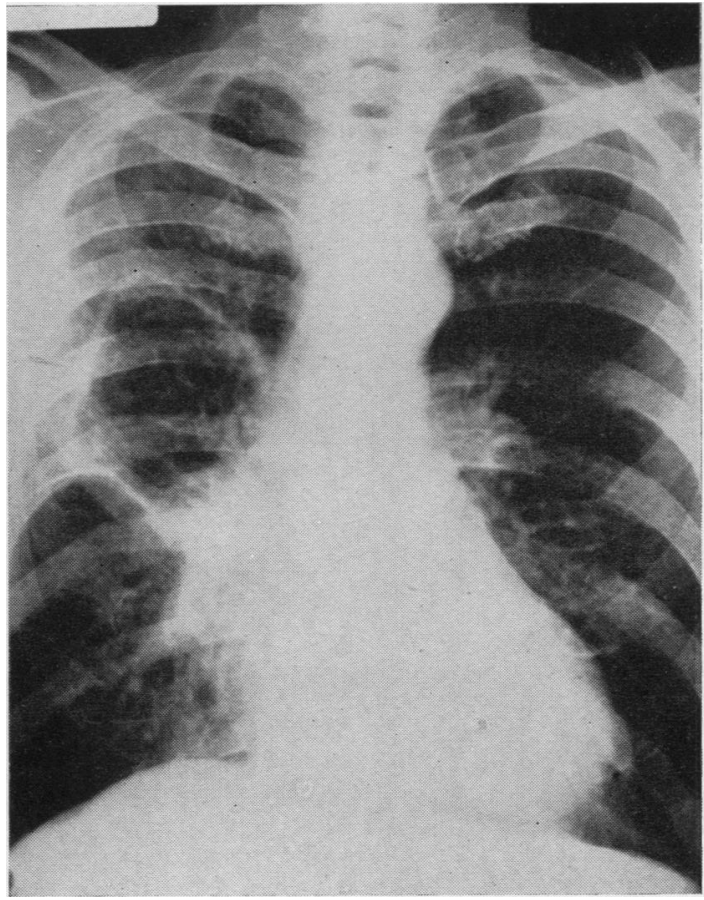

Fig. 2a.-Case 1: film taken in October, 1948, showing enlargement of cavity, without evidence of invasion or consolidation.

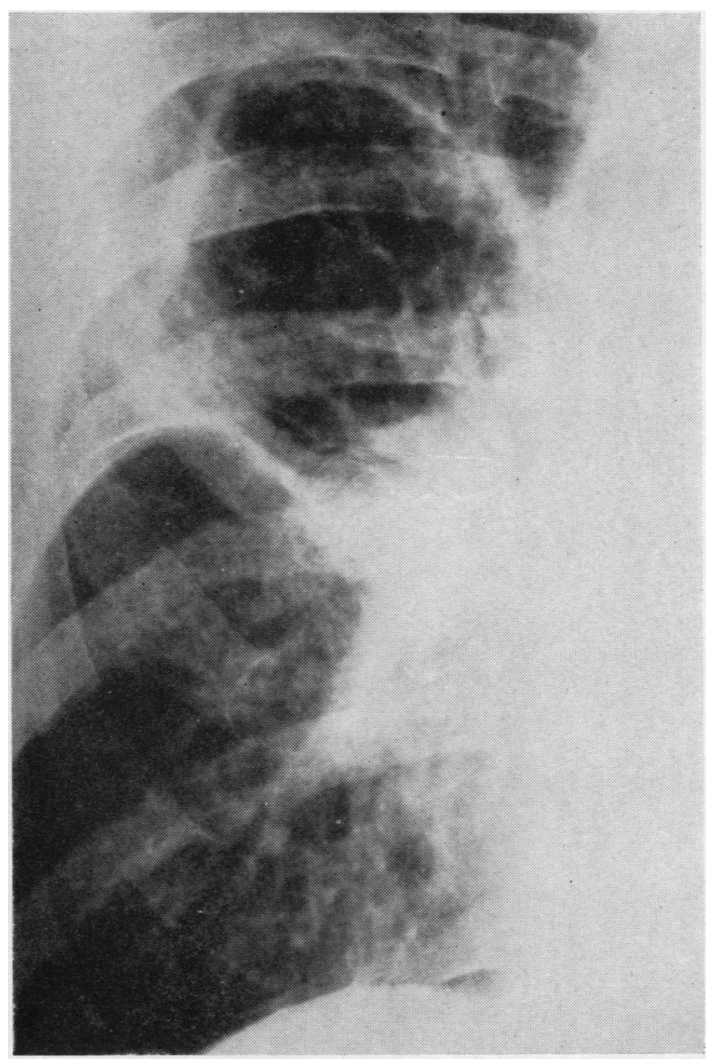

FIG. 2b.-Case 1: detailed vieiv of Fig. 2 a. 
FIG. 3a.-Case 2: radiograph showing thin-walled cavity in left upper lobe. There is also mediastinal and hilar glandular enlargement, and secondary inflammatory consolidation in the mid and lower zones.

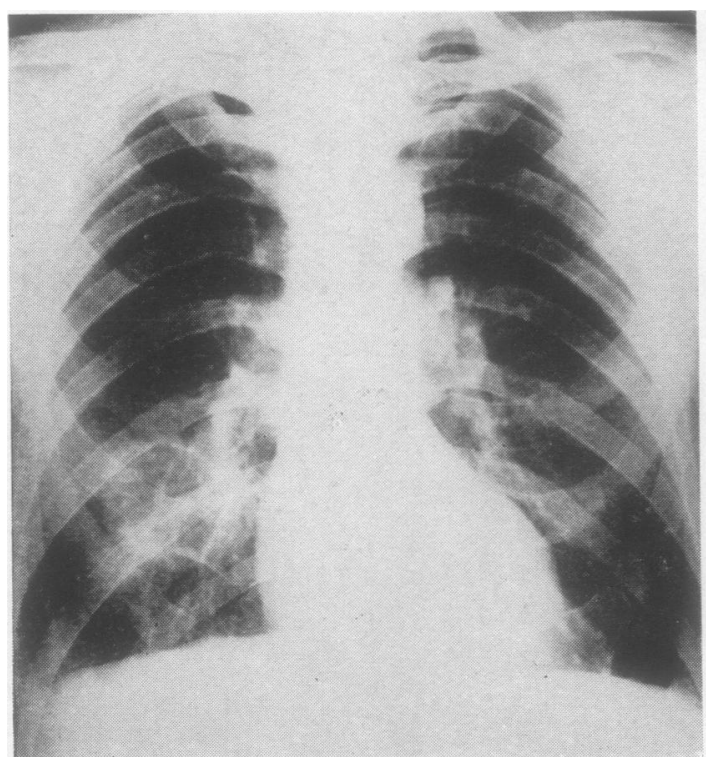

FIG, 4.-Case 3: thin-walled cavity in right lower lobe. Film taken in September, 1952.

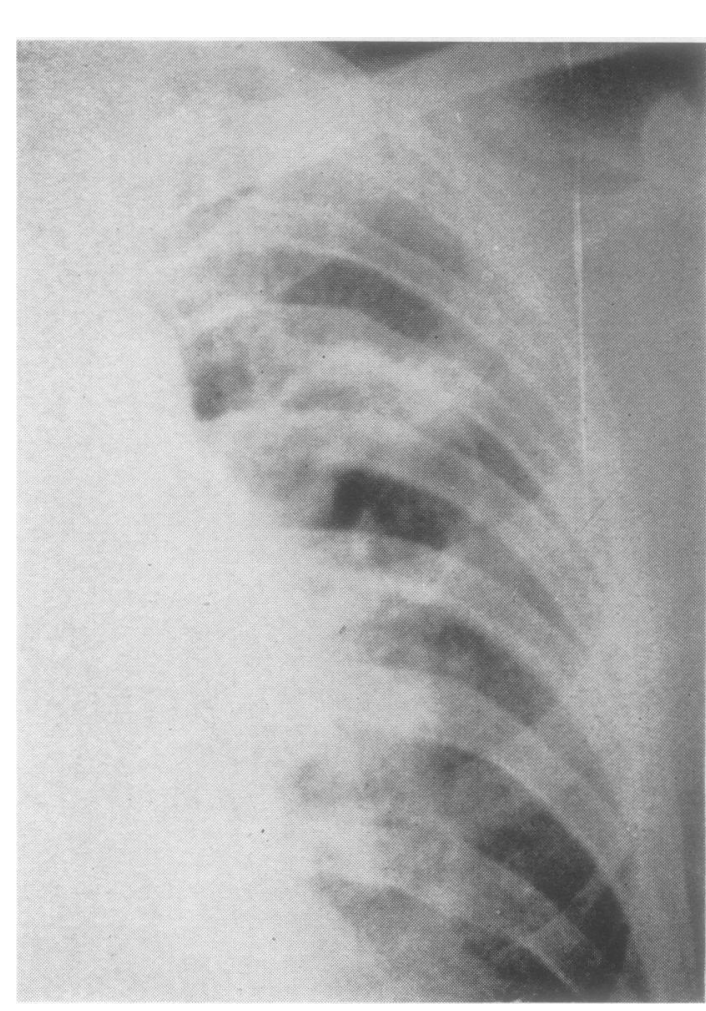

FIG. 3b.-Case 2: detailed view of Fig. 3a.

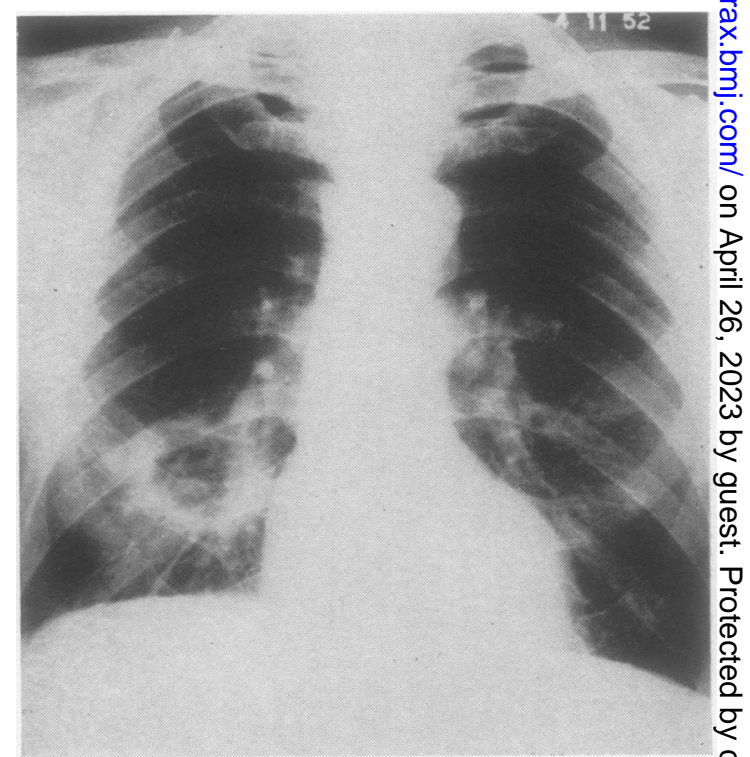

FIG. 5.-Case 3: film taken in November, 1952, showing that the cavity is smaller. 
Fig. 6a.-Case 4 : cavity in right lower lobe.

FIG. 6b.-Case 4: detailed view of Fig. 6a.

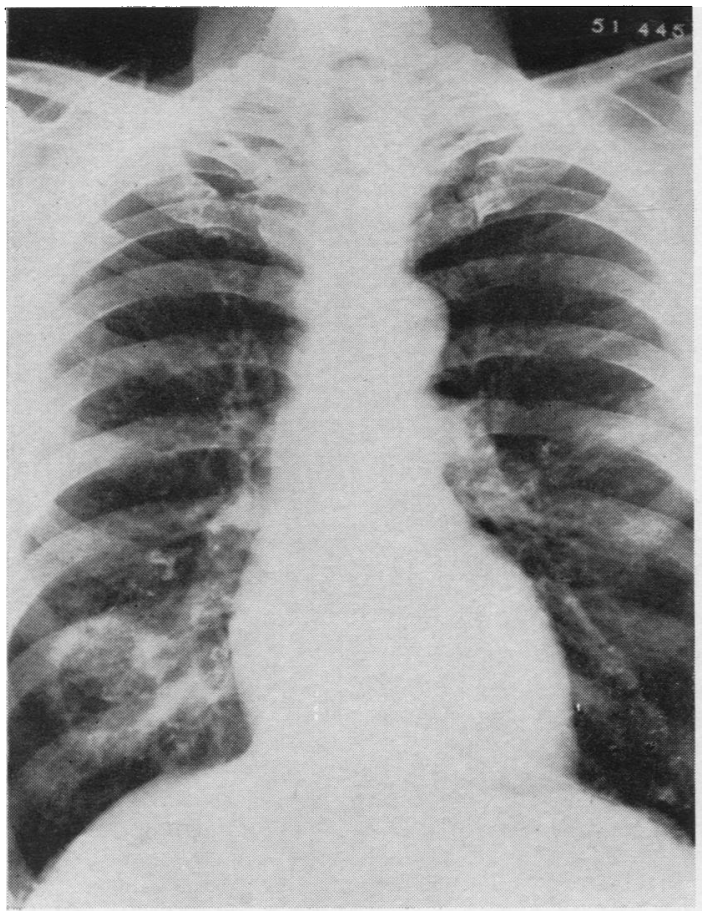

FIG. 6a.

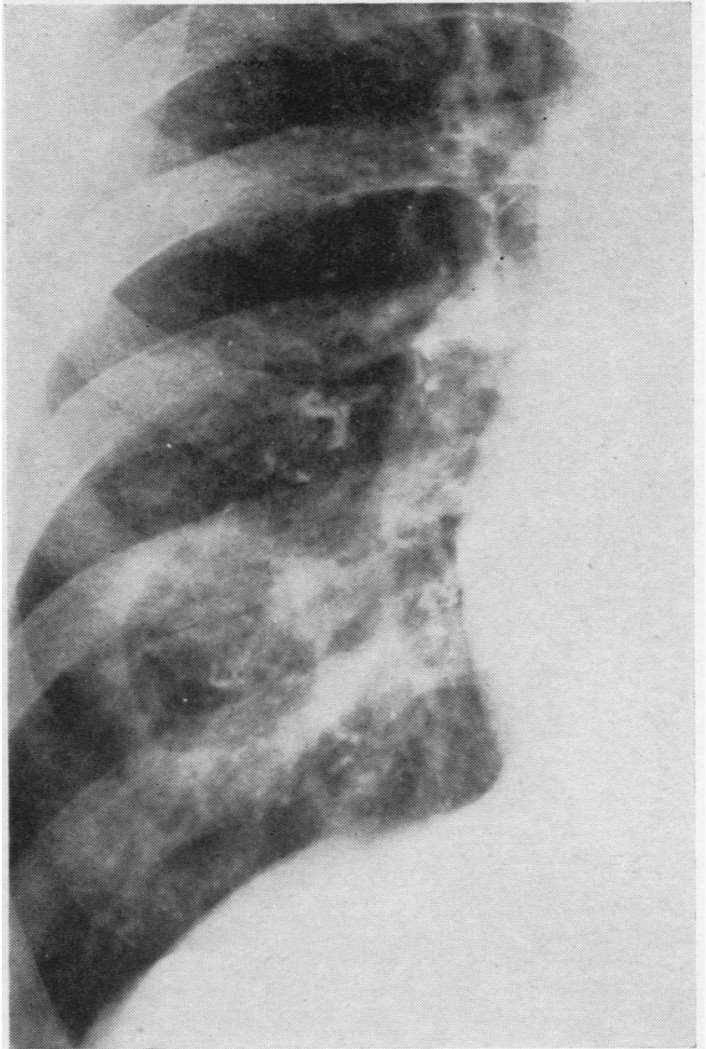

FiG. 6b.

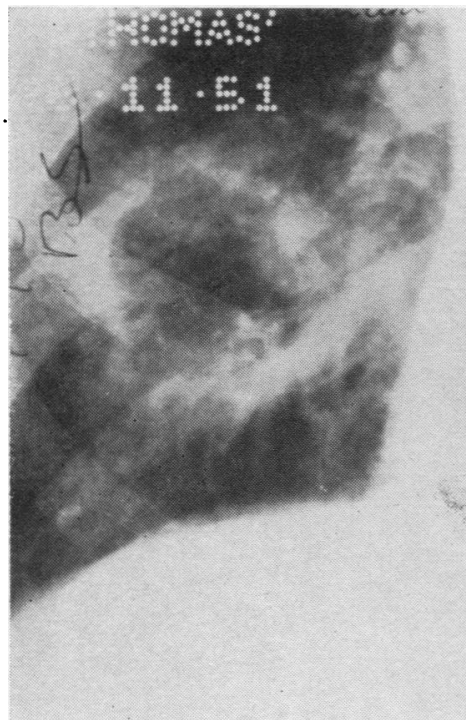

MID.RESPIRATION

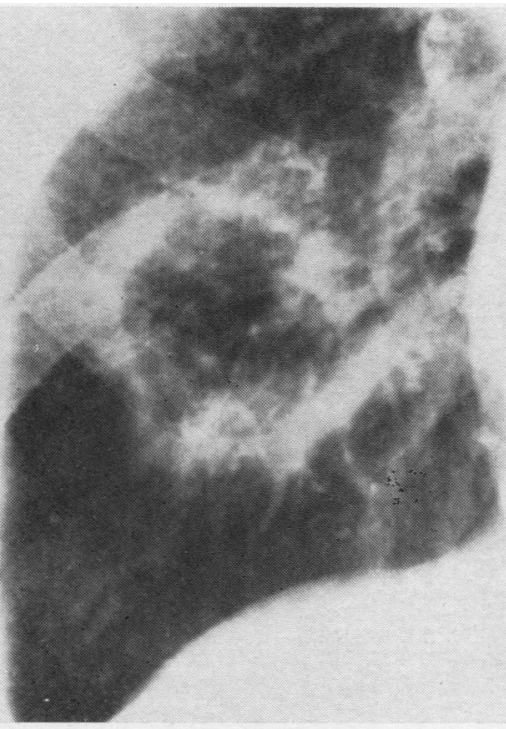

FULL INSPIRATION

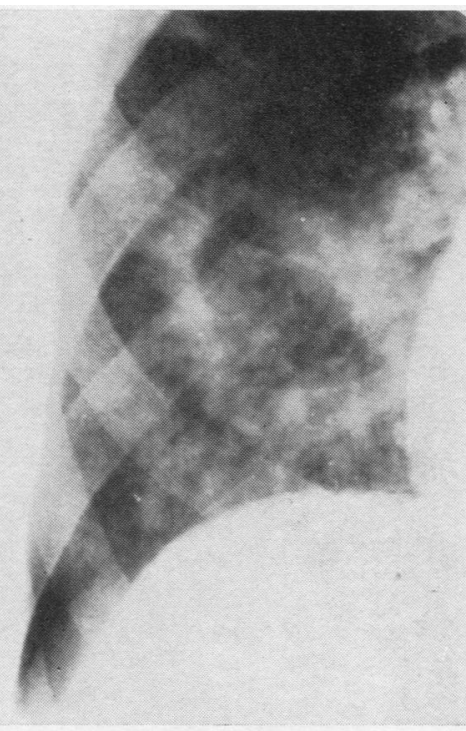

FULL EXPIRATION 


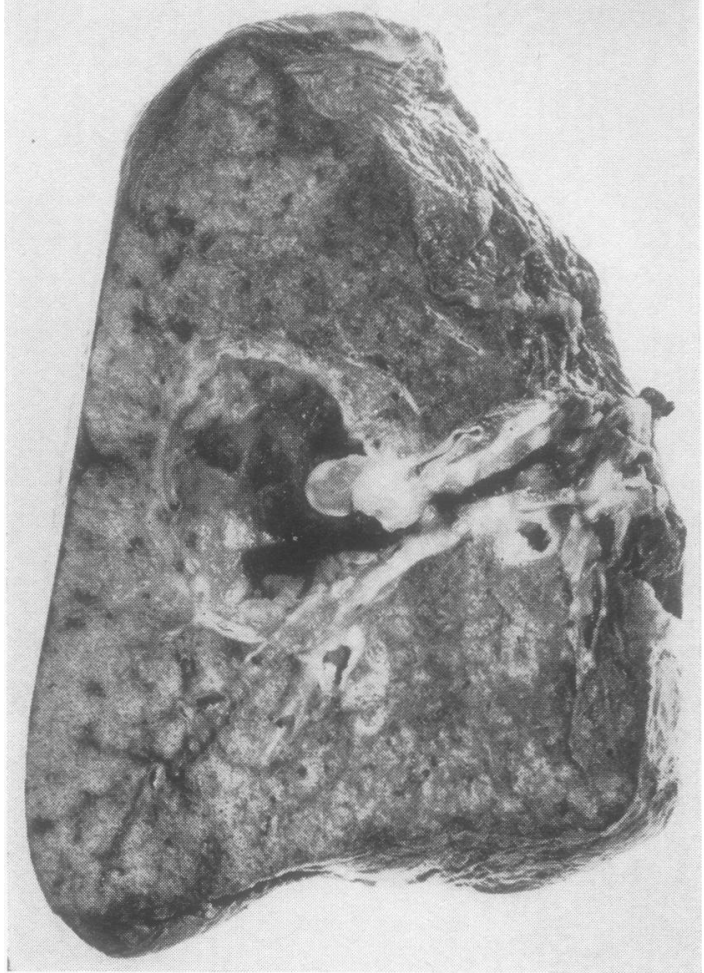

Fig. 8.-Case 4: photograph of the right lower lobe removed at operation sectioned to show a thin-walled cavity in relation to a bronchus with a nodule of growth at the entrance of bronchus into the cavity.

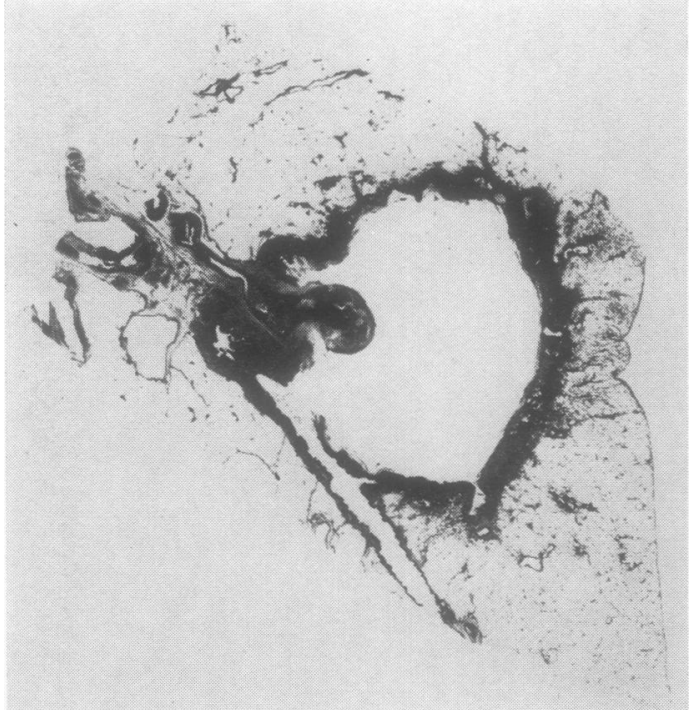

Fig. 9.-Case 4: print made direct from large section, showing a thin wall with smooth inner surface and absence of surrounding consolidation.

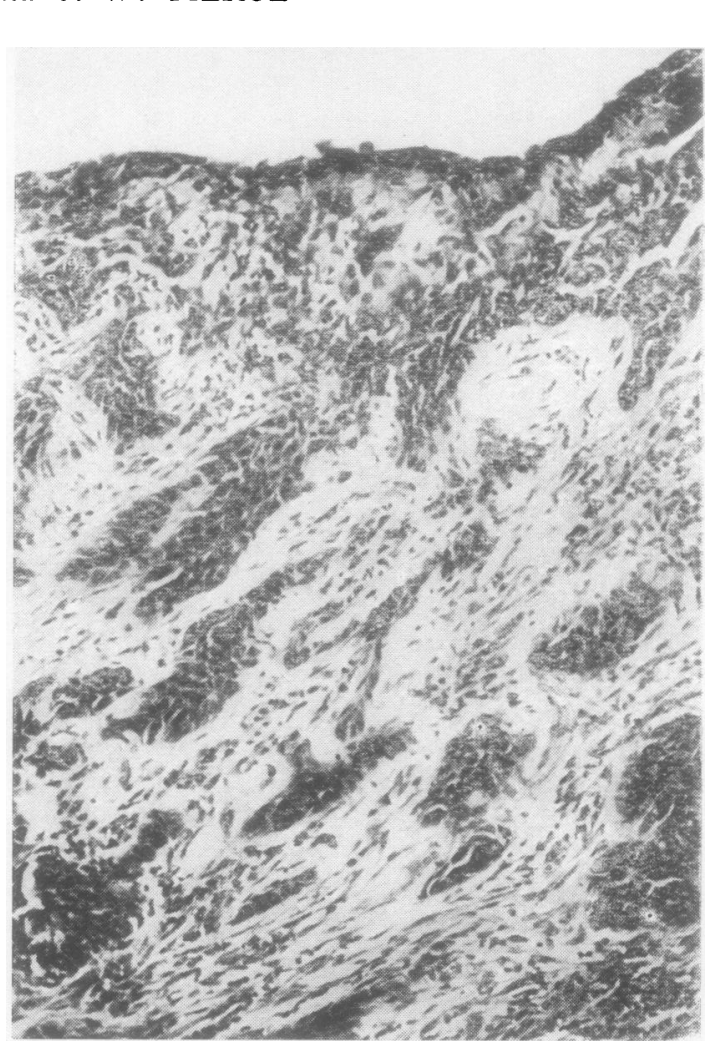

FIG. 10.-Case 4 : photomicrograph of the wall of the cavity showing a smooth regular layer of epithelial cells lining cavity, and absence of inflammatory or necrotic changes. Bulk of section composed of a mixture of fibrous tissue and squamous carcinoma cells.

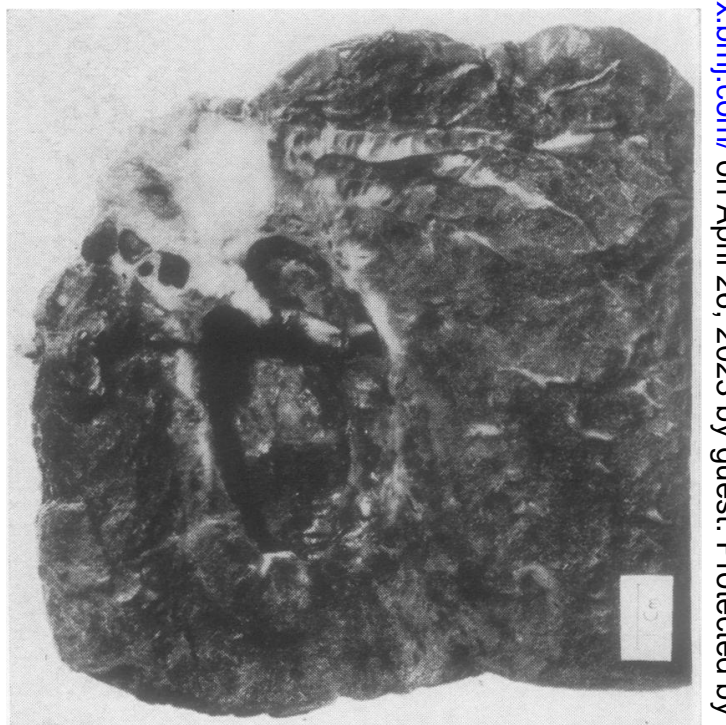

Fig. 11. Case 3: portion of right lower lobe removed at operation showing a thin-walled cavity with a smooth lining and no surrounding inflammation. 
histological findings that, first, the inner surface of these cavities shows remarkably little evidence of inflammation ; secondly, that the lining of malignant tissue is sometimes very thin and incomplete ; thirdly, by the occasional demonstration of the continuity between the cells in the cavity wall and a small nodule of growth in an adjacent bronchus ; and lastly, by the finding of metaplastic non-malignant epithelium lining the cyst wall with malignant cells (Fig. 10) beneath. (This we believe to be conclusive.) Finally it is difficult to explain the manner of their growth on the assumption that this takes place in two stages, an initial infiltration with later excavation, as no evidence of either infiltration or necrosis could be found.

\section{SUMMARY}

Carcinoma of the bronchus may occasionally present as a thin-walled cyst.

In each case the growth has been of the squamous-celled variety.

Reasons are given for believing that these cysts do not arise by the breakdown of solid growths, and an alternative hypothesis is suggested. 